\title{
ON SUBMANIFOLDS WITH TAMED SECOND FUNDAMENTAL FORM
}

\author{
G. PACELLI BESSA \\ Universidade Federal do Ceara, Brazil \\ e-mail: gpbessa@yahoo.com.br \\ and M. SILVANA COSTA \\ Universidade Federal do Ceara, Brazil \\ e-mail: silvana_math@yahoo.com.br
}

(Received 16 May 2008; revised 24 October 2008; accepted 15 May 2009)

\begin{abstract}
Based on the ideas of Bessa, Jorge and Montenegro (Comm. Anal. Geom., vol. 15, no. 4, 2007, pp. 725-732) we show that a complete submanifold $M$ with tamed second fundamental form in a complete Riemannian manifold $N$ with sectional curvature $K_{N} \leq \kappa \leq 0$ is proper (compact if $N$ is compact). In addition, if $N$ is Hadamard, then $M$ has finite topology. We also show that the fundamental tone is an obstruction for a Riemannian manifold to be realised as submanifold with tamed second fundamental form of a Hadamard manifold with sectional curvature bounded below.
\end{abstract}

1. Introduction. Let $\varphi: M \hookrightarrow N$ be an isometric immersion of a complete Riemannian $m$-manifold $M$ into a complete Riemannian $n$-manifold $N$ with sectional curvature $K_{N} \leq \kappa \leq 0$. Fix a point $x_{0} \in M$, and let $\rho_{M}(x)=\operatorname{dist}_{M}\left(x_{0}, x\right)$ be the distance function on $M$ to $x_{0}$. Let $\left\{C_{i}\right\}_{i=1}^{\infty}$ be an exhaustion sequence of $M$ by compact sets with $x_{0} \in C_{0}$. Let $\left\{a_{i}\right\} \subset[0, \infty]$ be a non-increasing sequence of possibly extended numbers defined by

$$
a_{i}=\sup \left\{\frac{S_{\kappa}}{C_{\kappa}}\left(\rho_{M}(x)\right) \cdot\|\alpha(x)\|, x \in M \backslash C_{i}\right\},
$$

where

$$
S_{\kappa}(t)=\left\{\begin{array}{cl}
\frac{1}{\sqrt{-\kappa}} \sinh (\sqrt{-\kappa} t) & \text { if } \quad \kappa<0 \\
t & \text { if } \quad \kappa=0
\end{array}\right.
$$

$C_{\kappa}(t)=S_{\kappa}^{\prime}(t)$ and $\|\alpha(x)\|$ is the norm of the second fundamental form at $\varphi(x)$. The number $a(M)=\lim _{i \rightarrow \infty} a_{i}$ does not depend on the exhaustion sequence $\left\{C_{i}\right\}$ nor on the base point $x_{0}$.

Definition 1.1. An immersion $\varphi: M \hookrightarrow N$ of a complete Riemannian $m$ manifold $M$ into an $n$-manifold $N$ with sectional curvature $K_{N} \leq \kappa \leq 0$ has tamed second fundamental form if $a(M)<1$.

In [4], Bessa, Jorge and Montenegro showed that a complete submanifold $\varphi: M \hookrightarrow$ $\mathbb{R}^{n}$ with tamed second fundamental form is proper and has finite topology, where finite 
topology means that $M$ is $C^{\infty}$-diffeomorphic to a compact smooth manifold $\bar{M}$ with boundary. In this paper we show that the ideas of Bessa, Jorge and Montenegro can be adapted to show that a complete submanifold $M \hookrightarrow N$ with tamed second fundamental form is proper. In addition if $N$ is a Hadamard manifold, then $M$ has finite topology. We prove the following theorem.

THEOREM 1.2. Let $\varphi: M \hookrightarrow N$ be an isometric immersion of a complete m-manifold $M$ into complete Riemannian n-manifold $N$ with sectional curvature $K_{N} \leq \kappa \leq 0$. Suppose that $M$ has tamed second fundamental form. Then

(a) $M$ is compact if $N$ is compact;

(b) $\varphi$ is proper if $N$ is non-compact;

(c) $M$ has finite topology if $N$ is a Hadamard manifold.

REMARK 1.3. Jorge and Meeks [10] showed that any complete $m$-dimensional submanifold $M$ of $\mathbb{R}^{n}$ homeomorphic to a compact Riemannian manifold $\bar{M}$, punctured at finite number of points $\left\{p_{1}, \ldots, p_{r}\right\}$ and having a well-defined normal vector at infinity has $a(M)=0$. This class of submanifolds includes all the complete minimal surfaces $\varphi: M^{2} \hookrightarrow \mathbb{R}^{n}$ with finite total curvature $\int_{M}|K|<\infty$ studied by Chern and Osserman $[7,14]$, all the complete surfaces $\varphi: M^{2} \hookrightarrow \mathbb{R}^{n}$ with finite total scalar curvature $\int_{M}|\alpha|^{2} d V<\infty$ and non-positive curvature with respect to every normal direction studied by White [16] and the $m$-dimensional minimal submanifolds $\varphi: M^{m} \hookrightarrow \mathbb{R}^{n}$ with finite total scalar curvature $\int_{M}|\alpha|^{m} d V<\infty$ studied by Anderson [1]. In [13], G. Oliveira Filho proved a version of Anderson's theorem for complete minimal submanifolds of $\mathbb{R}^{n}$ with finite total curvature $\int_{M}|\alpha|^{m} d V<\infty$.

Our second result shows that the fundamental tone $\lambda^{*}(M)$ can be an obstruction for a Riemannian manifold $M$ to be realised as a submanifold with tamed second fundamental form in a Hadamard manifold with bounded sectional curvature. The fundamental tone of a Riemannian manifold $M$ is given by

$$
\lambda^{*}(M)=\inf \left\{\frac{\int_{M}|\operatorname{grad} f|^{2}}{\int_{M} f^{2}}, f \in H_{0}^{1}(M) \backslash\{0\}\right\},
$$

where $H_{0}^{1}(M)$ is the completion of $C_{0}^{\infty}(M)$ with respect to the norm $|f|^{2}=\int_{M} f^{2}+$ $\int_{M}|\operatorname{grad} f|^{2}$. We prove the following theorem.

THEOREM 1.4. Let $\varphi: M \hookrightarrow N$ be an isometric immersion of a complete m-manifold $M$ with $a(M)<1$ into a Hadamard n-manifold $N$ with sectional curvature $\mu \leq K_{N} \leq$ 0 . Given $c, a(M)<c<1$, there exists $l=l(m, c) \in \mathbb{Z}_{+}$and a positive constant $C=$ $C(m, c, \mu)$ such that

$$
\lambda^{*}(M) \leq C \cdot \lambda^{*}\left(\mathbb{N}^{l}(\mu)\right)=C \cdot(l-1)^{2} \mu^{2} / 4,
$$

where $\mathbb{N}^{l}(\mu)$ is the l-dimensional simply connected space form of sectional curvature $\mu$.

REMARK 1.5. As corollary of Theorem (1.4) we have that $\lambda^{*}(M)=0$ for any submanifold $M$ mentioned in this list above.

Question 1.5. It is known [3, 5] that the fundamental tones of the Nadirashvilli bounded minimal surfaces [12] and the Martin-Morales cylindrically bounded minimal surfaces [11] are positive. We ask if there is a complete properly immersed (minimal) submanifold of the $\mathbb{R}^{n}$ with positive fundamental tone $\lambda^{*}>0$. 
2. Preliminaries. Let $\varphi: M \hookrightarrow N$ be an isometric immersion, where $M$ and $N$ are complete Riemannian manifolds. Consider a smooth function $g: N \rightarrow \mathbb{R}$ and the composition $f=g \circ \varphi: M \rightarrow \mathbb{R}$. Identifying $X$ with $d \varphi(X)$ we have at $q \in M$ and for every $X \in T_{q} M$ that

$$
\langle\operatorname{grad} f, X\rangle=d f(X)=d g(X)=\langle\operatorname{grad} g, X\rangle .
$$

Hence we write

$$
\operatorname{grad} g=\operatorname{grad} f+(\operatorname{grad} g)^{\perp},
$$

where $(\operatorname{grad} g)^{\perp}$ is perpendicular to $T_{q} M$. Let $\nabla$ and $\bar{\nabla}$ be the Riemannian connections on $M$ and $N$ respectively, and let $\alpha(x)(X, Y)$ and $\operatorname{Hess} f(x)(X, X)$ be respectively the second fundamental form of the immersion $\varphi$ and the Hessian of $f$ at $x$ with $X, Y \in$ $T_{x} M$. Using the Gauss equation we have that

$$
\operatorname{Hess} f(x)(X, Y)=\operatorname{Hess} g(\varphi(x))(X, Y)+\langle\operatorname{grad} g, \alpha(X, Y)\rangle_{\varphi(x)} .
$$

Taking the trace in (4), with respect to an orthonormal basis $\left\{e_{1}, \ldots, e_{m}\right\}$ for $T_{x} M$, we have that

$$
\begin{aligned}
\Delta f(x) & =\sum_{i=1}^{m} \operatorname{Hess} f(q)\left(e_{i}, e_{i}\right) \\
& =\sum_{i=1}^{m} \operatorname{Hess} g(\varphi(x))\left(e_{i}, e_{i}\right)+\left\langle\operatorname{grad} g, \sum_{i=1}^{m} \alpha\left(e_{i}, e_{i}\right)\right\rangle .
\end{aligned}
$$

We should mention that formulas (4) and (5) first appeared in [9]. If $g=h \circ \rho_{N}$, where $h: \mathbb{R} \rightarrow \mathbb{R}$ is a smooth function and $\rho_{N}$ is the distance function to a fixed point in $N$, then equation (4) becomes

$$
\operatorname{Hess} f(x)(X, X)=h^{\prime \prime}\left(\rho_{N}\right)\left\langle\operatorname{grad} \rho_{N}, X\right\rangle^{2}+h^{\prime}\left(\rho_{N}\right)\left[\operatorname{Hess} \rho_{N}(X, X)+\left\langle\operatorname{grad} \rho_{N}, \alpha(X, X)\right\rangle\right] \text {. }
$$

Another important tool in this paper the Hessian comparison theorem (see [9] or [15]).

THEOREM 2.1 Hessian comparison theorem. Let $N$ be a complete Riemannian $n$ manifold and $y_{0}, y \in N$. Let $\gamma:\left[0, \rho_{N}(y)\right] \rightarrow N$ be a minimising geodesic joining $y_{0}$ and $y$, where $\rho_{N}$ is the distance function to $y_{0}$ on $N$. Let $K_{\gamma}$ be the sectional curvatures of $N$ along $\gamma$. Denote by $\mu=\inf K_{\gamma}$ and $\kappa=\sup K_{\gamma}$. Then for all $X \in T_{y} N, X \perp \gamma^{\prime}\left(\rho_{N}(y)\right)$ the Hessian of $\rho_{N}$ at $y=\gamma\left(\rho_{N}(y)\right)$, satisfies

$$
\frac{C_{\mu}}{S_{\mu}}\left(\rho_{N}(y)\right)\|X\|^{2} \geq \operatorname{Hess} \rho_{N}(y)(X, X) \geq \frac{C_{\kappa}}{S_{\kappa}}\left(\rho_{N}(y)\right)\|X\|^{2},
$$

where $\operatorname{Hess} \rho_{N}(y)\left(\gamma^{\prime}, \gamma^{\prime}\right)=0$.

Observation 2.2. If $y \in \operatorname{cut}_{N}\left(y_{0}\right)$, inequality (7) has to be understood in the following sense:

$$
\frac{C_{\mu}}{S_{\mu}}\left(\rho_{N}(y)\right)\|X\|^{2} \geq \lim _{j \rightarrow \infty} \operatorname{Hess} \rho_{N}\left(y_{j}\right)\left(X_{j}, X_{j}\right) \geq \frac{C_{\kappa}}{S_{\kappa}}\left(\rho_{N}(y)\right)\|X\|^{2} .
$$

For a sequence $\left(y_{j}, X_{j}\right) \rightarrow(y, X) \in T N, y_{j} \notin \operatorname{cut}_{N}\left(y_{0}\right)$. 


\section{Proof of Theorem 1.2.}

3.1. Proof of items (a) and (b). Since $a(M)<1$, we have that for each $a(M)<$ $c<1$, there is $i$ such that $a_{i} \in(a(M), c)$. This means that there exists a geodesic ball $B_{M}\left(r_{0}\right) \subset M$, with $C_{i} \subset B_{M}\left(r_{0}\right)$, centred at $x_{0}$ with radius $r_{0}>0$ such that

$$
\frac{S_{\kappa}}{C_{\kappa}}\left(\rho_{M}(x)\right) \cdot\|\alpha(x)\| \leq c<1, \quad \text { for all } x \in M \backslash B_{M}\left(r_{0}\right) .
$$

To fix the notation, let $x_{0} \in M, y_{0}=\varphi\left(x_{0}\right)$ and $\rho_{M}(x)=\operatorname{dist}_{M}\left(x_{0}, x\right)$ and $\rho_{N}(y)=$ $\operatorname{dist}_{N}\left(y_{0}, y\right)$. Suppose first that $\kappa=0$. Letting $h(t)=t^{2}$ we have that $f(x)=\rho_{N}(\varphi(x))^{2}$. By equation (6) the Hessian of $f$ at $x \in M$ in the direction $X$ is given by

$$
\operatorname{Hess} f(x)(X, X)=2\left[\rho_{N} \operatorname{Hess} \rho_{N}(X, X)+\rho_{N}\left\langle\operatorname{grad} \rho_{N}, \alpha(X, X)\right\rangle+\left\langle\operatorname{grad} \rho_{N}, X\right\rangle^{2}\right](y),
$$

where $y=\varphi(x)$. By the Hessian comparison theorem, we have that

$$
\operatorname{Hess} \rho_{N}(y)(X, X) \geq \frac{1}{\rho_{N}(y)}\left\|X^{\perp}\right\|^{2},
$$

where $\left\langle X^{\perp}, \operatorname{grad} \rho_{N}\right\rangle=0$. Therefore for every $x \in M \backslash B_{M}\left(r_{0}\right)$,

$$
\begin{aligned}
\operatorname{Hess} f(x)(X, X)= & 2\left[\rho_{N} \operatorname{Hess} \rho_{N}(X, X)+\left\langle\operatorname{grad} \rho_{N}, X\right\rangle^{2}\right. \\
& \left.+\rho_{N}\left\langle\operatorname{grad} \rho_{N}, \alpha(X, X)\right\rangle\right](y) \\
\geq & 2\left[\rho_{N} \frac{1}{\rho_{N}}\left\|X^{\perp}\right\|^{2}+\left\|X^{\top}\right\|^{2}+\rho_{N}\left\langle\operatorname{grad} \rho_{N}, \alpha(X, X)\right\rangle\right](y) \\
\geq & 2\left[\left\|X^{\top}\right\|^{2}+\left\|X^{\perp}\right\|^{2}-\rho_{M}\|\alpha\| \cdot\|X\|^{2}\right] \\
\geq & 2(1-c)\|X\|^{2} .
\end{aligned}
$$

In the third and fourth lines of (11) we have used $\rho_{N}(\varphi(x)) \leq \rho_{M}(x)$. If $\kappa<0$, we let $h(t)=\cosh (\sqrt{-\kappa} t)$; then $f(x)=\cosh \left(\sqrt{-\kappa} \rho_{N}\right)(\varphi(x))$. By equation (6) the Hessian of $f$ is given by

$$
\begin{aligned}
\operatorname{Hess} f(x)(X, X)= & {\left[-\kappa \cosh \left(\sqrt{-\kappa} \rho_{N}\right)\left\langle\operatorname{grad} \rho_{N}, X\right\rangle^{2}+\sqrt{-\kappa} \sinh \left(\sqrt{-\kappa} \rho_{N}\right)\right.} \\
& \left.\times \operatorname{Hess} \rho_{N}(X, X)+\sqrt{-\kappa} \sinh \left(\sqrt{-\kappa} \rho_{N}\right)\left\langle\operatorname{grad} \rho_{N}, \alpha(X, X)\right\rangle\right](\varphi(x)) .
\end{aligned}
$$

By Hessian comparison theorem we have that

$$
\operatorname{Hess} \rho_{N}(y)(X, X) \geq \sqrt{-\kappa} \frac{\cosh \left(\sqrt{-\kappa} \rho_{N}\right)}{\sinh \left(\sqrt{-\kappa} \rho_{N}\right)}\left\|X^{\perp}\right\|^{2} .
$$

Since $a(M)<1$, we have

$$
\|\alpha(x)\| \leq c \sqrt{-\kappa} \frac{\cosh \left(\sqrt{-\kappa} \rho_{M}\right)}{\sinh \left(\sqrt{-\kappa} \rho_{M}\right)}(x) \leq c \sqrt{-\kappa} \frac{\cosh \left(\sqrt{-\kappa} \rho_{N}\right)}{\sinh \left(\sqrt{-\kappa} \rho_{N}\right.}(\varphi(x))
$$

for every $x \in M \backslash B_{M}\left(r_{0}\right)$ and some $c \in(0,1)$. The last inequality follows from the fact that $\rho_{N}(\varphi(x)) \leq \rho_{M}(x)$ and that the function $\sqrt{-\kappa} \operatorname{coth}(\sqrt{-\kappa} t)$ is non-increasing. 
Substituting in equation (12), we obtain

$$
\begin{aligned}
\operatorname{Hess} f(x)(X, X) \geq & -\kappa \cosh \left(\sqrt{-\kappa} \rho_{N}\right)\left\|X^{\perp}\right\|^{2}-\kappa \cosh \left(\sqrt{-\kappa} \rho_{N}\right)\left\|X^{\top}\right\|^{2} \\
& +\kappa \cdot c \cdot \cosh \left(\sqrt{-\kappa} \rho_{N}\right)\|X\|^{2} \\
\geq & -\kappa \cdot \cosh \left(\rho_{N}\right)(1-c)\|X\|^{2} \\
\geq & -\kappa \cdot(1-c) \cdot\|X\|^{2} .
\end{aligned}
$$

Let $\sigma:\left[0, \rho_{M}(x)\right] \rightarrow M$ be a minimal geodesic joining $x_{0}$ to $x$. For all $t>r_{0}$ we have that $(f \circ \sigma)^{\prime \prime}(t)=\operatorname{Hess} f(\sigma(t))\left(\sigma^{\prime}, \sigma^{\prime}\right) \geq 2(1-c)$ if $\kappa=0$ and $(f \circ \sigma)^{\prime \prime}(t) \geq-\kappa(1-c)$ if $\kappa<0$.

For $t \leq r_{0}$ we have that $(f \circ \sigma)^{\prime \prime}(t) \geq b=\inf \left\{\operatorname{Hess} f(x)(v, v), x \in B_{M}\left(r_{0}\right),|v|=1\right\}$. Hence $(\kappa=0)$,

$$
\begin{aligned}
(f \circ \sigma)^{\prime}(s) & =(f \circ \sigma)^{\prime}(0)+\int_{0}^{s}(f \circ \sigma)^{\prime \prime}(\tau) d \tau \\
& \geq(f \circ \sigma)^{\prime}(0)+\int_{0}^{r_{0}} b d \tau+\int_{r_{0}}^{s} 2(1-c) d \tau \\
& \geq(f \circ \sigma)^{\prime}(0)+b r_{0}+2(1-c)\left(s-r_{0}\right) .
\end{aligned}
$$

Now, $\rho_{N}\left(\varphi\left(x_{0}\right)\right)=\operatorname{dist}_{N}\left(y_{0}, y_{0}\right)=0$; then $(f \circ \sigma)^{\prime}(0)=0$ and $f\left(x_{0}\right)=0$; therefore

$$
\begin{aligned}
f(x) & =\int_{0}^{\rho_{M}(x)}(f \circ \sigma)^{\prime}(s) d s \\
& \geq \int_{0}^{\rho_{M}(x)}\left\{b r_{0}+2(1-c)\left(s-r_{0}\right)\right\} d s \\
& \geq b r_{0} \rho_{M}(x)+2(1-c)\left(\frac{\rho_{M}^{2}(x)}{2}-r_{0} \rho_{M}(x)\right) \\
& \geq(1-c) \rho_{M}^{2}(x)+(b-2(1-c)) r_{0} \rho_{M}(x) .
\end{aligned}
$$

Thus

$$
\rho_{N}^{2}(\varphi(x)) \geq(1-c) \rho_{M}^{2}(x)+(b-2(1-c)) r_{0} \rho_{M}(x)
$$

for all $x \in M$. Similarly, for $\kappa<0$ we obtain that

$$
\cosh \left(\sqrt{-\kappa} \rho_{N}\right)(\varphi(x)) \geq \sqrt{-\kappa}(1-c) \rho_{M}^{2}(x)+(b / \sqrt{-\kappa}-\sqrt{-\kappa}(1-c)) r_{0} \rho_{M}(x)+1 .
$$

If $N$ is compact, the left-hand sides of the inequalities (18) and (19) are bounded above. That implies that $M$ must be compact. In fact, we can find $\mu=\mu(\operatorname{diam}(N), c, \kappa)$ so that $\operatorname{diam}(M) \leq \mu$. Otherwise (if $N$ is complete non-compact) if $\rho_{M}(x) \rightarrow \infty$, then $\rho_{N}(\varphi) \rightarrow \infty$ and $\varphi$ is proper.

3.2. Proof of item (c). Recall that we have by hypothesis that $\varphi: M \hookrightarrow N$ is a complete $m$-dimensional submanifold with tamed second fundamental form immersed in complete $n$-dimensional Hadamard manifold $N$ with $K_{N} \leq \kappa \leq 0$. We can assume that $M$ is non-compact. Moreover, by item (a), proved in the last subsection, $\varphi$ is a proper immersion. Let $B_{N}\left(r_{0}\right)$ be the geodesic ball of $N$ centred at $y_{0}$ with radius $r_{0}$ 
and $S_{r_{0}}=\partial B_{N}\left(r_{0}\right)$. Since $\varphi$ is proper and $a(M)<1$ we can take $r_{0}$ so that

$$
\frac{S_{\kappa}}{C_{\kappa}}\left(\rho_{M}(x)\right)\|\alpha(x)\| \leq c<1, \quad \text { for all } x \in M \backslash \varphi^{-1}\left(B_{N}\left(r_{0}\right)\right),
$$

and by Sard's theorem (see [8], p. 79), $r_{0}$ can be chosen so that $\Gamma_{r_{0}}=\varphi(M) \cap S_{r_{0}} \neq \emptyset$ is a submanifold of $\operatorname{dim} \Gamma_{r_{0}}=m-1$. For each $y \in \Gamma_{r_{0}}$, let us denote by $T_{y} \Gamma_{r_{0}} \subset T_{y} \varphi(M)$ the tangent spaces of $\Gamma_{r_{0}}$ and $\varphi(M)$, respectively, at $y$. Since the dimension $\operatorname{dim} T_{y} \Gamma_{r_{0}}=$ $m-1$ and $\operatorname{dim} T_{y} \varphi(M)=m$, there exist only one unit vector $v(y) \in T_{y} \varphi(M)$ such that $T_{y} \varphi(M)=T_{y} \Gamma_{r_{0}} \oplus[[v(y)]]$, with $\left\langle v(y), \operatorname{grad} \rho_{N}(y)\right\rangle>0$. This defines a smooth vector field $v$ on a neighborhood $V$ of $\varphi^{-1}\left(\Gamma_{r_{0}}\right)$. Here $[[v(y)]]$ is the vector space generated by $v(y)$. Consider the function on $\varphi(V)$ defined by

$$
\psi(y)=\left\langle v, \operatorname{grad} \rho_{N}\right\rangle(y)=\langle v, \operatorname{grad} R\rangle(y)=v(y)(R), y=\varphi(x) .
$$

Then $\psi(y)=0$ if and only if every $x=\varphi^{-1}(y) \in V$ is a critical point of the extrinsic distance function $R$. Now for each $y \in \Gamma_{r_{0}}$ fixed, let us consider the solution $\xi(t, y)$ of the following Cauchy problem on $\varphi(M)$ :

$$
\left\{\begin{array}{l}
\xi_{t}(t, y)=\frac{1}{\psi} v(\xi(t, y)), \\
\xi(0, y)=y
\end{array}\right.
$$

We will prove that along the integral curve $t \mapsto \xi(t, y)$ there are no critical points for $R=\rho_{N} \circ \varphi$. For this, consider the function $(\psi \circ \xi)(t, y)$ and observe that

$$
\begin{aligned}
\psi_{t} & =\xi_{t}\left\langle\operatorname{grad} \rho_{N}, v\right\rangle \\
& =\left\langle\bar{\nabla}_{\xi_{t}} \operatorname{grad} \rho_{N}, v\right\rangle+\left\langle\operatorname{grad} \rho_{N}, \bar{\nabla}_{\xi_{t}} \nu\right\rangle \\
& =\frac{1}{\psi}\left\langle\bar{\nabla}_{\nu} \operatorname{grad} \rho_{N}, v\right\rangle+\frac{1}{\psi}\left\langle\operatorname{grad} \rho_{N}, \nabla_{\nu} v+\alpha(v, v)\right\rangle \\
& =\frac{1}{\psi} \operatorname{Hess} \rho_{N}(v, v)+\frac{1}{\psi}\left[\left\langle\operatorname{grad} \rho_{N}, \nabla_{\nu} v\right\rangle+\left\langle\operatorname{grad} \rho_{N}, \alpha(v, v)\right\rangle\right] \\
& =\frac{1}{\psi}\left[\operatorname{Hess} \rho_{N}(v, v)+\left\langle\operatorname{grad} \rho_{N}, \nabla_{\nu} v\right\rangle+\left\langle\operatorname{grad} \rho_{N}, \alpha(v, v)\right\rangle\right]
\end{aligned}
$$

Thus

$$
\psi_{t} \psi=\operatorname{Hess} \rho_{N}(v, v)+\left\langle\operatorname{grad} \rho_{N}, \nabla_{v} v\right\rangle+\left\langle\operatorname{grad} \rho_{N}, \alpha(v, v)\right\rangle
$$

Since $\langle v, v\rangle=1$, we have at once that $\left\langle\nabla_{v} v, v\right\rangle=0$. As $\nabla_{v} v \in T_{x} M$, we have that

$$
\left\langle\operatorname{grad} \rho_{N}, \nabla_{\nu} v\right\rangle=\left\langle\operatorname{grad} R, \nabla_{\nu} v\right\rangle .
$$

By equation (21), we can write $\operatorname{grad} R(x)=\psi(\varphi(x)) \cdot v(\varphi(x))$, since $\operatorname{grad} R(x) \perp$ $T_{\varphi(x)} \Gamma_{\rho_{N}(y)},\left(\Gamma_{\rho_{N}(y)}=\varphi(M) \cap \partial B_{N}\left(\rho_{N}(y)\right)\right)$. Then

$$
\left\langle\operatorname{grad} \rho_{N}, \nabla_{\nu} v\right\rangle=\left\langle\operatorname{grad} R, \nabla_{\nu} \nu\right\rangle=\psi\left\langle\nu, \nabla_{\nu} v\right\rangle=0 .
$$

Writing

$$
v(y)=\cos \beta(y) \operatorname{grad} \rho_{N}+\sin \beta(y) \omega
$$


and

$$
\operatorname{grad} \rho_{N}(y)=\cos \beta v(y)+\sin \beta v^{*},
$$

where $\left\langle\omega, \operatorname{grad} \rho_{N}\right\rangle=0$ and $\left\langle\nu, v^{*}\right\rangle=0$, equation (24) becomes

$$
\psi_{t} \psi=\sin ^{2} \beta \operatorname{Hess} \rho_{N}(\omega, \omega)+\sin \beta\left\langle v^{*}, \alpha(v, v)\right\rangle .
$$

From (25) we have that $\psi(y)=\cos \beta(y)$,

$$
\psi_{t} \psi=\sqrt{1-\psi^{2}} \sqrt{1-\psi^{2}} \operatorname{Hess} \rho_{N}(\omega, \omega)+\sqrt{1-\psi^{2}}\left\langle v^{*}, \alpha(v, v)\right\rangle .
$$

Hence

$$
\frac{\psi_{t} \psi}{\sqrt{1-\psi^{2}}}=\sqrt{1-\psi^{2}} \operatorname{Hess} \rho_{N}(\omega, \omega)+\left\langle v^{*}, \alpha(v, v)\right\rangle
$$

Thus we arrive at the following differential equation:

$$
-\left(\sqrt{1-\psi^{2}}\right)_{t}=\sqrt{1-\psi^{2}} \operatorname{Hess} \rho_{N}(\omega, \omega)+\left\langle v^{*}, \alpha(v, v)\right\rangle .
$$

The Hessian comparison theorem implies that

$$
\operatorname{Hess} \rho_{N}(\omega, \omega) \geq \frac{C_{\kappa}}{S_{\kappa}}\left(\rho_{N}(\xi(t, y))\right)
$$

Substituting it in equation (30) the following inequality is obtained:

$$
-\left(\sqrt{1-\psi^{2}}\right)_{t} \geq \sqrt{1-\psi^{2}} \frac{C_{\kappa}}{S_{\kappa}}\left(\rho_{N}(\xi(t, y))\right)+\left\langle v^{*}, \alpha(v, v)\right\rangle .
$$

Denoting by $R(t, y)$ the restriction of $R=\rho_{N} \circ \varphi$ to $\varphi^{-1}(\xi(t, y))$ we have

$$
R(t, y)=R\left(\varphi^{-1}(\xi(t, y))\right)=\rho_{N}(\xi(t, y)) .
$$

On the other hand we have that

$$
R_{t}=\left\langle\operatorname{grad} R, \frac{1}{\psi} v\right\rangle=\left\langle\psi v, \frac{1}{\psi} v\right\rangle=1 ;
$$

then

$$
R(t, y)=t+r_{0}
$$

Writing $\frac{C_{k}}{S_{k}}\left(\rho_{N}(\xi(t, y))\right)=\frac{C_{k}}{S_{k}}\left(t+r_{0}\right)$ in (32) we have

$$
-\left(\sqrt{1-\psi^{2}}\right)_{t} \geq \sqrt{1-\psi^{2}} \frac{C_{\kappa}}{S_{\kappa}}\left(t+r_{0}\right)+\left\langle v^{*}, \alpha(v, v)\right\rangle .
$$

Multiplying (35) by $S_{\kappa}\left(t+r_{0}\right)$, the following is obtained:

$$
-\left[S_{\kappa}\left(t+r_{0}\right)\left(\sqrt{1-\psi^{2}}\right)_{t}+C_{\kappa}\left(t+r_{0}\right) \sqrt{1-\psi^{2}}\right] \geq S_{k}\left(t+r_{0}\right)\left\langle v^{*}, \alpha(v, v)\right\rangle .
$$


The last inequality can be written as

$$
\left[S_{\kappa}\left(t+r_{0}\right) \sqrt{1-\psi^{2}}\right]_{t} \leq-S_{\kappa}\left(t+r_{0}\right)\left\langle v^{*}, \alpha(v, v)\right\rangle .
$$

Integrating (36) from 0 to $t$ the resulting inequality is as follows:

$$
S_{\kappa}\left(t+r_{0}\right) \sin \beta(\xi(t, y)) \leq S_{\kappa}\left(r_{0}\right) \sin \beta(y)+\int_{0}^{t}-S_{k}\left(s+r_{0}\right)\left\langle v^{*}, \alpha(v, v)\right\rangle d s .
$$

Thus

$$
\sin \beta(\xi(t, y)) \leq \frac{S_{\kappa}\left(r_{0}\right)}{S_{k}\left(t+r_{0}\right)} \sin \beta(y)+\frac{1}{S_{\kappa}\left(t+r_{0}\right)} \int_{0}^{t} S_{\kappa}\left(s+r_{0}\right)\left(-\left\langle v^{*}, \alpha(v, v)\right\rangle\right) d s .
$$

Since $a(M)<1$,

$$
\begin{aligned}
-\left\langle v^{*}, \alpha(v, v)\right\rangle(\xi(s, y)) & \leq\|\alpha(\xi(s, y))\| \leq c \frac{C_{\kappa}}{S_{\kappa}}\left(\rho_{M}(\xi(s, y))\right) \\
& \leq c \frac{C_{\kappa}}{S_{\kappa}}\left(\rho_{N}(\xi(s, y))\right)=c \frac{C_{\kappa}}{S_{\kappa}}\left(s+r_{0}\right)
\end{aligned}
$$

for every $s \geq 0$. Substituting in (37), we have

$$
\begin{aligned}
\sin \beta(\xi(t, y)) & \leq \frac{S_{\kappa}\left(r_{0}\right)}{S_{\kappa}\left(t+r_{0}\right)} \sin \beta(y)+\frac{c}{S_{\kappa}\left(t+r_{0}\right)} \int_{0}^{t} C_{\kappa}\left(s+r_{0}\right) d s \\
& =\frac{S_{\kappa}\left(r_{0}\right)}{S_{\kappa}\left(t+r_{0}\right)} \sin \beta(y)+\frac{c}{S_{\kappa}\left(t+r_{0}\right)}\left(S_{\kappa}\left(t+r_{0}\right)-S_{\kappa}\left(r_{0}\right)\right) \\
& =\frac{S_{\kappa}\left(r_{0}\right)}{S_{\kappa}\left(t+r_{0}\right)}(\sin \beta(y)-c)+c<1
\end{aligned}
$$

for all $t \geq 0$. Therefore, along the integral curve $t \mapsto \xi(t, y)$, there are no critical points for the function $R(x)=\rho_{N}(\varphi(x))$ outside the geodesic ball $B_{N}\left(r_{0}\right)$. The flow $\xi_{t}$ maps $\partial B_{N}\left(r_{0}\right)$ diffeomorphically into $\partial B_{N}\left(r_{0}+t\right)$, for all $t \geq 0$. This shows that $M$ has finite topology (see also [6]). This concludes the proof of Theorem 1.2. For the sake of clarity we show that $\frac{S_{\kappa}\left(r_{0}\right)}{S_{\kappa}\left(t+r_{0}\right)}(\sin \beta(y)-c)+c<1$. Let $h(t)=\frac{S_{\kappa}\left(r_{0}\right)}{S_{\kappa}\left(t+r_{0}\right)}(\sin \beta(y)-c)+c$. We have that $h(0)=\sin \beta<1$ and $h^{\prime}(t)=-\frac{C_{\kappa}\left(t+r_{0}\right) S_{\kappa}\left(r_{0}\right)}{\left.S_{\kappa}^{2}\left(t+r_{0}\right)\right)}(\sin \beta-c)$. If $\sin \beta \geq c$, then $h^{\prime}(t) \leq 0$ and $h(t) \leq h(0)$. If $\sin \beta<c$, suppose by contradiction that there exists a $T>0$ such that $h(T)>1$. This implies that $0>S_{\kappa}\left(r_{0}\right)(\sin \beta-c)>(1-c) S_{\kappa}\left(T+r_{0}\right)>0$.

4. Proof of Theorem 1.4. The first ingredient for the proof of Theorem 1.4 is the well-known Barta's theorem [2] stated here for the sake of completeness.

THEOREM 4.1 (Barta). Let $\Omega$ be a bounded open of a Riemannian manifold with piecewise smooth boundary. Let $f \in C^{2}(\Omega) \cap C^{0}(\bar{\Omega})$ with $f \mid \Omega>0$ and $f \mid \partial \Omega=0$. The first Dirichlet eigenvalue $\lambda_{1}(\Omega)$ has the following bounds:

$$
\sup _{\Omega}\left(-\frac{\Delta f}{f}\right) \geq \lambda_{1}(\Omega) \geq \inf _{\Omega}\left(\frac{-\Delta f}{f}\right),
$$

with equality in (4) if and only iff is the first eigenfunction of $\Omega$. 
Let $\varphi: M \hookrightarrow N$ be an isometric immersion with tamed second fundamental form of a complete $m$-manifold $M$ into a Hadamard $n$-manifold $N$ with sectional curvature $\mu \leq K_{N} \leq 0$. Let $x_{0} \in M, y_{0}=\varphi\left(x_{0}\right) \in N$, and let $\rho_{N}(y)=\operatorname{dist}_{N}\left(y_{0}, y\right)$ be the distance function on $N$ and $\rho_{N} \circ \varphi$ the extrinsic distance on $M$. By the proof of Theorem (1.2) there is an $r_{0}>0$ such that there is no critical point $x \in M \backslash \varphi^{-1}\left(B_{N}\left(r_{0}\right)\right)$ for $\rho_{N} \circ \varphi$, where $B_{N}\left(r_{0}\right)$ is the geodesic ball in $N$ centred at $y_{0}$ with radius $r_{0}$. Let $R>r_{0}$, and let $\Omega \subset \varphi^{-1}\left(B_{N}(R)\right)$ be a connected component. Since $\varphi$ is proper we have that $\Omega$ is bounded with boundary $\partial \Omega$ that we may suppose to be piecewise smooth. Let $v: B_{\mathbb{N}^{\prime}(\mu)}(R) \rightarrow \mathbb{R}$ be a positive first eigenfunction of the geodesic ball of radius $R$ in the $l$-dimensional simply connected space form $\mathbb{N}^{l}(\mu)$ of constant sectional curvature $\mu$, where $l$ is to be determined. The function $v$ is radial, i.e. $v(x)=v(|x|)$, and satisfies the following differential equation:

$$
v^{\prime \prime}(t)+(l-1) \frac{C_{\mu}}{S_{\mu}}(t) v^{\prime}(t)+\lambda_{1}\left(B_{\mathbb{N}^{\prime}(\mu)}(R)\right) v(t)=0, \quad \forall t \in[0, R],
$$

with initial data $v(0)=1, v^{\prime}(0)=0$. Moreover, $v^{\prime}(t)<0$ for all $t \in(0, R] ; S_{\mu}$ and $C_{\mu}$ are defined in (1) and $\lambda_{1}\left(B_{\mathbb{N}^{\prime}(\mu)}(R)\right)$ is the first Dirichlet eigenvalue of the geodesic ball $B_{\mathbb{N}^{\prime}(\mu)}(R) \subset \mathbb{N}^{l}(\mu)$ with radius $R$. Define $\tilde{v}: B_{N}(R) \rightarrow \mathbb{R}$ by $\tilde{v}(y)=v \circ \rho_{N}(y)$ and $f: \Omega \rightarrow \mathbb{R}$ by $f(x)=\tilde{v} \circ \varphi(x)$. By Barta's theorem we have $\lambda_{1}(\Omega) \leq \sup _{\Omega}(-\triangle f / f)$. The Laplacian $\triangle f$ at a point $x \in M$ is given by

$$
\begin{aligned}
\triangle_{M} f(x) & =\left[\sum_{i=1}^{m} \operatorname{Hess} \tilde{v}\left(e_{i}, e_{i}\right)+\langle\operatorname{grad} \tilde{v}, \vec{H}\rangle\right](\varphi(x)) \\
& =\sum_{i=1}^{m}\left[v^{\prime \prime}\left(\rho_{N}\right)\left\langle\operatorname{grad} \rho_{N}, e_{i}\right\rangle^{2}+v^{\prime}\left(\rho_{N}\right) \operatorname{Hess} \rho_{N}\left(e_{i}, e_{i}\right)\right]+v^{\prime}(\rho)\left\langle\operatorname{grad} \rho_{N}, \vec{H}\right\rangle,
\end{aligned}
$$

where Hess $\tilde{v}$ is the Hessian of $\tilde{v}$ in the metric of $N$ and $\left\{e_{i}\right\}_{i=1}^{m}$ is an orthonormal basis for $T_{x} M$ at which we made the identification $\varphi_{*} e_{i}=e_{i}$. We are going to give an upper bound for $(-\triangle f / f)$ on $\varphi^{-1}\left(B_{N}(R)\right)$. Let $x \in \varphi^{-1}\left(B_{N}(R)\right)$, and choose an orthonormal basis $\left\{e_{1}, \ldots, e_{m}\right\}$ for $T_{x} M$ such that $\left\{e_{2}, \ldots, e_{m}\right\}$ are tangent to the distance sphere $\partial B_{N}(r(x))$ of radius $r(x)=\rho_{N}(\varphi(x))$ and $e_{1}=\left\langle e_{1}, \operatorname{grad}_{N} \bar{\rho}\right\rangle \operatorname{grad}_{N} \bar{\rho}+\left\langle e_{1}, \partial / \partial \theta\right\rangle \partial / \partial \theta$, where $|\partial / \partial \theta|=1, \partial / \partial \theta \perp \operatorname{grad}_{N} \bar{\rho}$. To simplify the notation set $t=\rho_{N}(\varphi(x)), \triangle_{M}=\triangle$. Then

$$
\begin{aligned}
\Delta f(x)= & \sum_{i=1}^{m}\left[v^{\prime \prime}(t)\left\langle\operatorname{grad} \rho_{N}, e_{i}\right\rangle^{2}+v^{\prime}(t) \operatorname{Hess} \rho_{N}\left(e_{i}, e_{i}\right)\right]+v^{\prime}(t)\left\langle\operatorname{grad} \rho_{N}, \vec{H}\right\rangle \\
= & v^{\prime \prime}(t)\left\langle\operatorname{grad} \rho_{N}, e_{1}\right\rangle^{2}+v^{\prime}(t)\left\langle e_{1}, \partial / \partial \theta\right\rangle^{2} \operatorname{Hess} \rho_{N}(\partial / \partial \theta, \partial / \partial \theta) \\
& +\sum_{i=2}^{m} v^{\prime}(t) \operatorname{Hess} \rho_{N}\left(e_{i}, e_{i}\right)+v^{\prime}(t)\left\langle\operatorname{grad} \rho_{N}, \vec{H}\right\rangle .
\end{aligned}
$$

Thus from (41)

$$
\begin{aligned}
-\frac{\Delta f}{f}(x)= & -\frac{v^{\prime \prime}}{v}(t)\left\langle\operatorname{grad} \rho_{N}, e_{1}\right\rangle^{2}-\frac{v^{\prime}}{v}(t)\left\langle e_{1}, \partial / \partial \theta\right\rangle^{2} \operatorname{Hess} \rho_{N}(\partial / \partial \theta, \partial / \partial \theta) \\
& -\sum_{i=2}^{m} \frac{v^{\prime}}{v}(t) \operatorname{Hess} \rho_{N}\left(e_{i}, e_{i}\right)-\frac{v}{v}^{\prime}(t)\left\langle\operatorname{grad} \rho_{N}, \vec{H}\right\rangle .
\end{aligned}
$$


Equation (40) says that

$$
-\frac{v^{\prime \prime}}{v}(t)=(l-1) \frac{C_{\mu}}{S_{\mu}} \frac{v^{\prime}}{v}(t)+\lambda_{1}\left(B_{\mathbb{N}^{\prime}(\mu)}(R)\right) .
$$

By the Hessian comparison theorem and the fact $v^{\prime} / v \leq 0$ we have from equation (42) the following inequality:

$$
\begin{aligned}
-\frac{\triangle f}{f}(x) \leq & \left.\lambda_{1}\left(B_{\mathbb{N}^{\prime}(\mu)}(R)\right)\right]\left[1-\left\langle e_{1}, \partial / \partial \theta\right\rangle^{2}\right] \\
& -\frac{C_{\mu}}{S_{\mu}}(t) \frac{v^{\prime}}{v}(t)\left[m-l+l\left\langle e_{1}, \partial / \partial \theta\right\rangle^{2}+\frac{S_{\mu}}{C_{\mu}}\|\vec{H}\|\right] .
\end{aligned}
$$

On the other hand the mean curvature vector $\vec{H}$ at $\varphi(x)$ has the norm

$$
\|\vec{H}\|(\varphi(x)) \leq\|\alpha\|(\varphi(x)) \leq c \cdot\left(C_{\kappa} / S_{\kappa}\right)\left(\rho_{M}(x)\right) \leq c \cdot\left(C_{\kappa} / S_{\kappa}\right)\left(\rho_{N}(\varphi(x))\right) .
$$

We have that for any given $a(M)<c<1$ there exist $r_{0}=r_{0}(c)>0$ such that there is no critical point $x \in M \backslash \varphi^{-1}\left(B_{N}\left(r_{0}\right)\right)$ for $\rho_{N} \circ \varphi$. A critical point $x$ satisfies $\left\langle e_{1}, \partial / \partial \theta\right\rangle(\varphi(x))=1$ (see equation (25), where $\left\langle e_{1}, \partial / \partial \theta\right\rangle(\varphi(x))=\sin \beta(\varphi(x))$ ). Inequality (38) shows that for any $x \in M \backslash \varphi^{-1}\left(B_{N}\left(r_{0}\right)\right)$ we have $(\kappa=0$ in our case)

$$
\begin{aligned}
\left\langle e_{1}, \partial / \partial \theta\right\rangle(\varphi(x)) & \left.\leq \frac{r_{0}}{\rho_{N}(\varphi(x))+r_{0}}\left(\sup _{z \in \varphi^{-1}\left(\partial B_{N}\left(r_{0}\right)\right)} \sin \beta(\varphi(z))\right)-c\right)+c \\
& \leq \frac{r_{0}}{r_{0}+r_{0}}(1-c)+c \\
& =\frac{1+c}{2} .
\end{aligned}
$$

We have then from (43) and (44) the following inequality:

$$
-\frac{\triangle f}{f}(x) \leq \lambda_{1}\left(B_{\mathbb{N}^{\prime}(\mu)}(R)\right)-\frac{C_{\mu}}{S_{\mu}}(t) \frac{v^{\prime}}{v}(t)\left[m-l+\frac{l}{4}(1+c)^{2}+c\right] .
$$

Choose the least $l \in \mathbb{Z}_{+}$such that $m-l+l(1+c)^{2} / 4+c \leq 0$. With this choice of $l$ we have for all $x \in \varphi^{-1}\left(B_{N}(R) \backslash B_{N}\left(r_{0}\right)\right)$ that

$$
-\frac{\triangle f}{f}(x) \leq \lambda_{1}\left(B_{\mathbb{N}^{\prime}(\mu)}(R)\right) .
$$

Now let $x \in \varphi^{-1}\left(B_{N}\left(r_{0}\right)\right)$. Since $1-\left\langle e_{1}, \partial / \partial \theta\right\rangle^{2} \leq 1$ and $-l+l\left\langle e_{1}, \partial / \partial \theta\right\rangle^{2} \leq 0$ we obtain from (43) the following inequality $\left(t=\rho_{N}(\varphi(x))\right)$ :

$$
\left.-\frac{\triangle f}{f}(x) \leq \lambda_{1}\left(B_{\mathbb{N}^{\prime}(\mu)}(R)\right)\right]-\frac{C_{\mu}}{S_{\mu}}(t) \frac{v^{\prime}}{v}(t)\left[m+\frac{S_{\mu}}{C_{\mu}}\|\vec{H}\|\right] .
$$

We need the following technical lemma.

LEMMA 4.2. Let $v$ be the function satisfying (40). Then $-v^{\prime}(t) / t \leq \lambda_{1}\left(B_{\mathbb{N}^{\prime}(\mu)}(R)\right)$ for all $t \in[0, R]$. 
Proof. Consider the function $h:[0, R] \rightarrow \mathbb{R}$ given by $h(t)=\lambda \cdot t+v^{\prime}(t), \lambda=$ $\lambda_{1}\left(B_{\mathbb{N}^{\prime}(\mu)}(R)\right)$. We know that $v(0)=1, v^{\prime}(0)=0$ and $v^{\prime}(t) \leq 0$; besides $v$ satisfies equation (40). Observe that

$$
0=v^{\prime \prime}(t)+(l-1) v^{\prime}+\lambda v \leq v^{\prime \prime}+\lambda .
$$

Thus $v^{\prime \prime} \geq-\lambda$ and $h^{\prime}(t)=\lambda+v^{\prime \prime} \geq 0$. Since $h(0)=0$ we have $h(t)=\lambda t+v^{\prime}(t) \geq 0$. This proves the lemma.

Since $v$ is a non-increasing positive function we have $v(t) \geq v\left(r_{0}\right)$. Applying Lemma (4.2) we obtain

$$
\begin{aligned}
-\frac{\Delta f}{f}(x) & \leq \lambda_{1}\left(B_{\mathbb{N}^{\prime}(\mu)}(R)\right)+\frac{t \cdot C_{\mu}(t)}{S_{\mu}(t)}\left(-\frac{v^{\prime}(t)}{t}\right) \cdot \frac{1}{v\left(r_{0}\right)}[m+c] \\
& \leq \lambda_{1}\left(B_{\mathbb{N}^{\prime}(\mu)}(R)\right)\left[1+r_{0} \frac{C_{\mu}}{S_{\mu}}\left(r_{0}\right) \cdot \frac{1}{v\left(r_{0}\right)}[m+c]\right] .
\end{aligned}
$$

Thus for all $x \in \varphi^{-1}\left(B_{N}(R)\right)$ we have

$$
\begin{aligned}
-(\Delta f / f)(x) & \leq \max \left\{1,\left[1+r_{0} \frac{C_{\mu}}{S_{\mu}}\left(r_{0}\right) \cdot \frac{1}{v\left(r_{0}\right)}[m+c]\right]\right\} \cdot \lambda_{1}\left(B_{\mathbb{N}^{\prime}(\mu)}(R)\right) \\
& =\left[1+r_{0} \frac{C_{\mu}}{S_{\mu}}\left(r_{0}\right) \cdot \frac{1}{v\left(r_{0}\right)}[m+c]\right] \cdot \lambda_{1}\left(B_{\mathbb{N}^{\prime}(\mu)}(R)\right) .
\end{aligned}
$$

Then by Barta's theorem

$$
\lambda_{1}(\Omega) \leq\left[1+r_{0} \frac{C_{\mu}}{S_{\mu}}\left(r_{0}\right) \cdot \frac{1}{v\left(r_{0}\right)}[m+c]\right] \cdot \lambda_{1}\left(B_{\mathbb{N}^{\prime}(\mu)}(R)\right) .
$$

Observe that $C=\left[1+r_{0} \frac{C_{\mu}}{S_{\mu}}\left(r_{0}\right) \cdot \frac{1}{v\left(r_{0}\right)}[m+c]\right]$ does not depend on $R$. So letting $R \rightarrow$ $\infty$ we have $\lambda^{*}(M) \leq C \lambda^{*}\left(\mathbb{N}^{l}(\mu)\right)$.

Corollary 4.3 (From the proof). Given c, $a(M)<c<1$, there exists $r_{0}=r_{0}(c)>$ $0, l=l(m, c) \in \mathbb{Z}_{+}$and $C=C(m, \mu, c)>0$ such that for any $R>r_{0}$ and any connected component $\Omega$ of $\varphi^{-1}\left(B_{N}(r)\right)$, then

$$
\lambda^{*}(\Omega) \leq C \cdot \lambda_{1}\left(B_{\mathbb{N}^{\prime}(\mu)}(R)\right) .
$$

\section{REFERENCES}

1. M. Anderson, The compactification of a minimal subamnifold by the Gauss map, Unpublished preprint (Institute Des Hautes Etudes Scientifiques (IHES), Bures-sur-Yvette, France, 1985). $472-473$.

2. J. Barta, Sur la vibration fundamental d'une membrane, C. R. Acad. Sci. 204 (1937),

3. G. P. Bessa and J. Fabio Montenegro, An extension of Barta's theorem and geometric applications, Ann. Global Anal. Geom. 31(4) (2007), 345-362.

4. G. P. Bessa, L. P. Jorge and J. Fabio Montenegro, Complete submanifolds of $\mathbb{R}^{n}$ with finite topology, Comm. Anal. Geom. 15(4) (2007), 725-732.

5. G. P. Bessa and M. Silvana Costa, Eigenvalue estimates for submanifolds with locally bounded mean curvature in $N \times \mathbb{R}$, Proc. Amer. Math. Soc. 137 (2009), 1093-1102. 
6. J. Cheeger, Critical points of distance functions and applications to geometry, in Geometric topology: Recent developments, Lecture Notes in Mathematics, vol. 1504 (Morel J.-M., Takens F. and Teissier B., Editors) (Springer, Berlin, 1991), 1-38.

7. S. S. Chern and R. Osserman, Complete minimal surfaces in euclidean $n$-space, J. d'Anal. Math. 19 (1967), 15-34.

8. B. A. Dubrovin, A. T. Fomenko and S. P. Novikov, Modern geometry-methods and applications. Part II. The geometry and topology of manifolds (Axler S. and Ribet K. A., Editors) (Graduate Texts in Mathematics, vol. 104, Springer-Verlag, New York, Heidelberg, Tokyo, 1985, ISBN 0-387-96162-3).

9. L. P. Jorge and D. Koutroufiotis, An estimative for the curvature of bounded submanifolds, Amer. J. Math. 103 (1981), 711-725.

10. L. P. Jorge and W. Meeks III, The topology of complete minimal surfaces of finite total Gaussian curvature, Topology 22 (1983), 203-221.

11. F. Martín and S. Morales, A complete bounded minimal cylinder in $\mathbb{R}^{3}$, Michigan Math. J. 47(3) (2000), 499-514.

12. N. Nadirashvili, Hadamard's and Calabi-Yau's conjectures on negatively curved and minimal surfaces, Invent. Math. 126 (1996), 457-465.

13. G. Oliveira Filho, Compactification of minimal submanifolds of hyperbolic space, Comm. Anal. Geom. 1(1) (1993), 1-29.

14. R. Osserman, Global properties of minimal surfaces in $\mathbb{E}^{3}$ and $\mathbb{E}^{n}$, Ann. Math. 80 (1964), 340-364.

15. R. Schoen and S. Yau, Lectures on differential geometry, Conference Proceedings and Lecture Notes in Geometry and Topology, vol. 1 (Yau S. T., Editor) (International Press, Somerville, MA, 1994).

16. B. White, Complete surfaces of finite total curvature, J. Diff. Geom. 26 (1987), 315-326. 